\title{
Ist die telemedizinische Versorgung von Patienten mit COPD sinnvoll?
}

\author{
Is the Telemedical Management of COPD Patients of any Value?
}

Autoren

Institute
U. Koehler ${ }^{1}$, O. Hildebrandt ${ }^{1}$, K. I. Koehler ${ }^{1}$, V. Gross ${ }^{2}$, A. Weissflog ${ }^{3}$, A. K. Sohrabi ${ }^{3}$

${ }^{1}$ Klinik für Innere Medizin; SP Pneumologie, Intensiv- und Schlafmedizin (Direktor: Prof. Dr. C. Vogelmeier), Philipps-Universität Marburg

${ }^{2}$ Technische Hochschule Mittelhessen, Fachbereich KMUB, Biomedizinische Technik, Gießen

${ }^{3}$ Thora Tech GmbH, Anwenderzentrum Medizintechnik, Gießen eingereicht $\quad$ 8.2.2012

akzeptiert 9.2.2012

\section{Bibliografie}

DOI http://dx.doi.org/

10.1055/s-0031-1291827

Online-Publikation: 4.4.2012

Pneumologie 2012; 66: 302-304

(c) Georg Thieme Verlag KG

Stuttgart · New York

ISSN 0934-8387

Korrespondenzadresse
Prof. Dr. Ulrich Koehler
Klinik für Innere Medizin
SP Pneumologie, Intensiv- und
Schlafmedizin
Universitätsklinikum Gießen
und Marburg GmbH
Baldingerstraße 1
35033 Marburg
koehleru@staff.uni-marburg.de

koehleru@staff.uni-marburg.de

\section{COPD - Sozioökonomische Relevanz \\ $\nabla$}

Berücksichtigt man den demografischen Wandel, die Zunahme älter werdender Menschen sowie den Anstieg chronischer Erkrankungen, wird klar, dass die Kosten im Gesundheitssystem zwangsläufig weiter steigen werden. Bereits heute sind etwa 75\% der Ausgaben im Gesundheitswesen durch chronisch kranke Patienten verursacht. Die enorme sozioökonomische Bedeutung der chronischen Lungenerkrankungen, insbesondere der COPD, ist unbestritten. Die Prävalenz der chronischen Bronchitis wird bei der erwachsenen Bevölkerung in Deutschland auf 10 bis $15 \%$ geschätzt. Weltweit ist die COPD gegenwärtig die vierthäufigste Todesursache. Für die nächsten Jahrzehnte ist von einem weiteren Anstieg von Prävalenz, Morbidität und Mortalität der COPD auszugehen. Die jährlichen volkswirtschaftlichen Gesamtkosten der COPD werden auf 5,9 Mrd.€, die individuellen Kosten eines Patienten auf $3027 € /$ Patient geschätzt. Krankenhausaufenthalte stellen den bedeutendsten Kostenfaktor dar [1]

\section{Telemedizin bei COPD - Was ist zu fordern?}

Neben der verbesserten Versorgung spielt vor allem die Kosteneffektivität angesichts der steigenden Zahlen chronisch kranker Menschen eine große Rolle. Ziele telemedizinischer Versorgung sollen eine umfassende Information, Betreuung und Steuerung der Patientenversorgung mithilfe von elektronischen Medien sein. Ein verbesserter Datenaustausch und die Möglichkeit eines kontinuierlichen Transfers von Gesundheitsinformationen sollen vor allem auch die Lebensqualität chronisch Kranker verbessern. Ergebnisse bisheriger Studien und Metaanalysen belegen zwar, dass es unter telemedizinischer Überwachung von Patienten mit COPD zu einer Reduktion von
Krankheitsexazerbationen sowie zu einer Reduktion von Notaufnahmebehandlungen und stationären Aufnahmen kommt, hinsichtlich der Verbesserung der Lebensqualitätsparameter sowie der Morbidität und Mortalität ist jedoch bislang kein relevanter Effekt nachweisbar [2 -5]. Problematisch bei den bislang vorliegenden Studien erscheint die Heterogenität der untersuchten Stichproben sowie die der angewandten Messmethoden und Zielkriterien. Eine telemedizinische Intervention kann jedoch nur dann erfolgreich sein, wenn klare Definitionen und Auswahlkriterien hinsichtlich der zu überwachenden Patienten, des überwachenden Personals sowie der Parameter der Messmethode gewährleistet werden. Was ist notwendig, um eine solche „ideale“ Kooperation zwischen Betreuendem und Betreutem zu erreichen?

- Die zu überwachenden Patienten müssen kritisch selektiert werden: Es sollten ausschließlich Patienten mit schwergradiger COPD der GOLD-Stadien III und IV berücksichtigt werden.

- Die Patienten müssen einen vertrauenswürdigen und medizinisch versierten Ansprechpartner haben.

- Es muss jederzeit ein schnelles Eingreifen/ Therapiemanagement möglich sein.

- Die medikamentöse und nichtmedikamentöse Therapie sollte leitliniengerecht sein.

- Das telemedizinische Verfahren sollte Schulungs- und Lernkomponenten beinhalten.

- Die derzeitigen „Informations-ÜbertragungsSysteme“ sind seitens der Messparameter (Herzfrequenz, Sauerstoffsättigung, PeakFlow) zu optimieren.

- Die Messparameter sollten nach Möglichkeit nicht nur punktuell, sondern im Langzeitverlauf erfassbar sein (z. B. Lungengeräusche; Sauerstoffsättigung). 
- Telemedizin kann auch bei der Überwachung der LOT und nichtinvasiven Beatmung sinnvoll eingesetzt werden.

- Mithilfe von Telemedizin kann die Durchführung häuslicher körperlicher Trainingsmaßnahmen supervidiert werden.

\section{Telemedizin bei Patienten mit COPD - Warum und bei wem sinnvoll? \\ $\nabla$}

Der Erfolg telemedizinischer Überwachung von COPD-Patienten ist wesentlich von der Patientenselektion abhängig $[2,3,5,6]$. So hat die kontinuierliche telemedizinische Versorgung von Asthmapatienten aller Altersgruppen weder eine Verbesserung der Lebensqualität noch eine Reduzierung der Notfallbehandlungen belegen können. Die Zahl der Klinikaufenthalte war rückläufig, insbesondere bei Patienten mit schwergradiger Erkrankung [4]. Die Rekrutierung von Patienten für den Lungensport hat gezeigt, dass insbesondere solche mit schwergradiger COPD Grad III und IV von den sporttherapeutischen Maßnahmen profitieren und eine sehr hohe Therapiecompliance aufweisen. Regelhaft wiederholte Wissensvermittlung trägt zudem zur verbesserten Compliance und auch zum verbesserten Selbstmanagement der Erkrankung bei. Der Patient lernt, vergleichbar dem Lungensport, sich symptombezogen und belastungsabhängig einzuschätzen. Exazerbationen können vom Patienten (und damit auch vom Betreuenden) frühzeitiger erkannt und therapiert werden. Dem Arzt-Patienten-Gespräch muss selbstverständlich weiterhin eine hohe Priorität eingeräumt werden. Ultimativ einzufordern ist eine menschliche und fachliche Kompetenz des überwachenden Personals. Nur die Vertrauensbeziehung zwischen den Betreuenden und den Betreuten vermag langfristig den Erfolg eines telemedizinischen Konzeptes zu gewährleisten. Die telemedizinische Versorgung muss beweisen, dass sie kritische Entwicklungen im Verlauf einer chronischen Erkrankung (Exazerbationen) frühzeitig erkennen kann, zumal Eigen- und Fachkompetenz zum richtigen Zeitpunkt abgerufen werden müssen. Ein großer Teil an stationären Aufnahmen könnte verhindert werden, wenn Patienten und Arzt rechtzeitig und adäquat auf die Symptome einer beginnenden Exazerbation reagieren würden. Um Eigenkompetenz zu fördern, reicht die telemedizinische Übermittlung von Vitalparametern aber nicht aus: Nur die dauerhafte Reflexion, das Vermitteln von Reaktionsmustern sowie die „Erziehung“ zum selbstständigen Patienten gewährleisten langfristig Autonomie und Therapieeffizienz.

\section{Telemedizin - auch bei der Überwachung von Sauer- stofflangzeittherapie und nichtinvasiver Ventilation sinnvoll \\ $\nabla$}

Da es sich bei der COPD um eine chronische, nicht reversible Erkrankung handelt, wird mit fortschreitendem Verlauf eine intensivere Betreuung und Behandlung der Patienten notwendig. Bei vielen Patienten mit schwergradiger COPD oder anderweitiger chronischer Lungenerkrankung sind eine Sauerstoffversorgung sowie eine Atmungsunterstützung (nichtinvasive Beatmung) erforderlich. Lebenserhaltende Medizintechnik wie Sauerstoffkonzentratoren, mobile Flüssigsauerstoffsysteme, Beatmungsgeräte und Therapiegeräte von schlafbezogenen Atemstörungen müssen regelmäßig technischen Kontrollen unterzogen werden, um ihre Funktionsfähigkeit zu gewährleisten. Gerade bei älteren Patienten kommt es bei der Anwendung der Geräte gehäuft zu Fehl- bedienungen und Fehleinschätzungen. So werden oft Funktionsdefekte am Gerät nicht oder zu spät dem Serviceunternehmen gemeldet. Dabei ergeben sich auch Probleme in der zeitnahen Bewertung und ggf. Instandsetzung der Geräte, da bei den meisten Patienten die Sauerstoffkonzentratoren bzw. LOX-Systeme während der Nachtstunden zum Einsatz kommen.

Das Ziel der Etablierung eines telemedizinischen Überwachungssystems wäre der Onlinenachweis der Gerätefunktionalität und Gerätesicherheit. Eine Ferndiagnose der Geräte kann technische Komplikationen schon im Vorfeld erkennen und dadurch die Versorgung der Patienten sicherer machen. Derzeit wird, in Zusammenarbeit von Technischer Hochschule Mittelhessen (THM), der Klinik für Pneumologie des Universitätsklinikums Gießen und Marburg GmbH sowie dem Ingenieurbüro für Medizintechnik aus Wettenberg (IfM GmbH), in einem vom Bundeswirtschaftsministerium geförderten Projekt ein Modul entwickelt, welches die kontinuierliche Messung der Geräteparameter im häuslichen Umfeld erlaubt und den zeitnahen Nachweis der Einhaltung sicherheitsrelevanter Parameter dokumentiert [7]. Bei abweichenden Messwerten wird mithilfe eines GSM-Moduls (Global System for Mobile Communications) per Textnachricht (SMS) eine $\mathrm{Zu}$ standsmeldung mit Fehlerbeschreibung an eine „virtuelle Leitstelle“ gesendet. Die virtuelle Leitstelle stellt eine intelligente Einheit dar, welche einen Bericht an den zuständigen Adressaten übermittelt (siehe $\bullet$ Abb. 1).

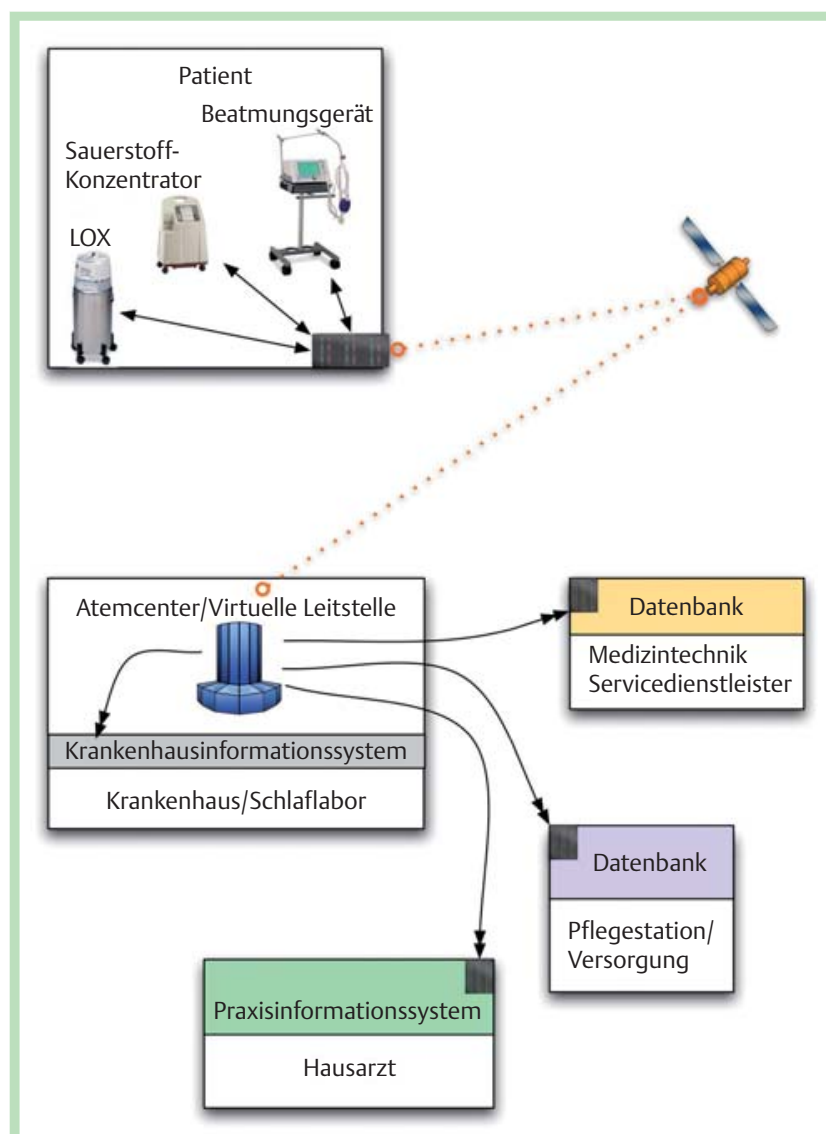

Abb. 1 Der Tele-Assistent für $\mathrm{O}_{2}$-Konzentratoren und LOX-Systeme in der Heimbeatmung [7]. Informationen von Geräten im Home-Care-Bereich werden an ein integriertes Funkmodul übermittelt, welches die Daten dann an die Empfangseinheit der Leitstelle sendet. Die Daten/Signale werden ausgewertet, gefiltert und intelligent zugeordnet. 
Technische Probleme werden im Servicebereich bei dem Servicedienstleister analysiert. Der technische Kundendienst kann gezielt auf das Problem eingehen und abwägen, inwiefern der Fehler vom Benutzer selbst behoben werden kann oder, ob und wann, eine Intervention vor Ort notwendig ist. Durch schnelles Handeln werden die Gerätesicherheit und die optimale Versorgung des Patienten gewährleistet, ohne dass der Patient sich mit technischen Fragen auseinandersetzen muss.

\section{Zusammenfassung}

Ob die telemedizinische Betreuung chronisch kranker Patienten mit respiratorischer Insuffizienz wirklich eine nachhaltige Verbesserung der Patientenversorgung sowie der Kostenstruktur mit sich bringen wird, ist derzeit noch spekulativ. Es ist zu mutmaßen, dass durch den Einsatz telemedizinischer Verfahren eine verbesserte Vernetzung von Leistungserbringern und -empfängern (Patienten) erzielt werden kann. Wichtig erscheinen die kritische Patientenselektion, die einheitliche Definition von Zielkriterien sowie eine effiziente Betreuung. Die Messparameter sollten überdacht (akustische Phänomene berücksichtigen!) und um eine zeitliche Dynamik ergänzt werden. Unter Berücksichtigung dieser Faktoren sollten eine verbesserte Versorgung der Patienten und damit eine Kostenreduktion möglich sein. Die Heterogenität der bislang untersuchten Stichproben erlaubt nur eine begrenzte Aussage bezüglich der Effizienz einer telemedizinischen Versorgung von Patienten mit COPD.

\section{Interessenkonflikt}

U. Koehler erhielt Honorare von AstraZeneca, GlaxoSmithKline, IfM, Heinen und Loewenstein; Unterstützung von Forschungsvorhaben durch AstraZeneca, GlaxoSmithKline, Berlin-Chemie, IfM, Heinen und Loewenstein, Resmed, Respironics, Weinmann, UCB Biosciences.

O. Hildebrandt, K. I. Koehler, V. Gross, A. Weissflog und A. K. Sohrabi geben an, dass kein Interessenkonflikt besteht.

\section{Literatur}

1 Vogelmeier C, Buhl R, Criée CP et al. Leitlinie der Deutschen Atemwegsliga und der deutschen Gesellschaft für Pneumologie und Beatmungsmedizin zur Diagnostik und Therapie von Patienten mit chronisch obstruktiver Bronchitis und Lungenemphysem (COPD). Pneumologie 2007; 61: e1-e40

2 Trappenburg J, Niesink A, Gerdien $H$ et al. Effects of telemonitoring in patients with chronic obstructive pulmonary disease. Telemedicine and e-health 2008; 14: 138-146

3 Smith SM, Brame A, Kulinskaya $E$ et al. Telemonitoring and Intermediate Care. Chest 2011; 139: $731-732$

4 McLean S, Chandler D, Nurmatov $U$ et al. Telehealthcare for asthma: a Cochrane review. CMAJ 2011; 183: 733-742

5 McLean S, Nurmatov U, Liu JLY et al. Telehealthcare for chronic obstructive pulmonary disease (Review). Cochrane Database of Systematic Reviews 2011; 7: 1-51

6 Vitacca M, Bianchi L, Guerra A et al. Tele-assistance in chronic respiratory failure patients: a randomised clinical trial. Eur Respir J 2009; 33: $411-418$

7 Sohrabi AK, Weissflog A, Leiche $O$ et al. Telemedizinische Versorgung chronischer Lungenerkrankungen. Mt-Medizintechnik 2011; 131: $216-219$ 